\title{
Vibration-assisted optical injection of a single fluorescent sensor into a target cell
}

\author{
Hengjun Liu*, Hisataka Maruyama, Taisuke Masuda, Fumihito Arai \\ Department of Micro-Nano Systems Engineering, Nagoya University, Nagoya 464-8603, Japan \\ Email: liuhj@biorobotics.mech.nagoya-u.ac.jp, hisataka@mech.nagoya-u.ac.jp, \\ masuda@mech.nagoya-u.ac.jp, arai@mech.nagoya-u.ac.jp, \\ *Corresponding author: liuhj@,biorobotics.mech.nagoya-u.ac.jp \\ Nagoya University, Furo-cho, Chikusa-ku, Nagoya, 464-8603, Japan \\ Tel: +81-52-789-5026, Fax: +81-52-789-5027
}

\begin{abstract}
In this paper, we propose the selective adhesion and rapid injection of a fluorescent sensor into a target cell via the optical control of zeta potential and local vibration stimulus using optical tweezers. A multi-fluorescent sensor, which can respond to both temperature and $\mathrm{pH}$, was encapsulated in anionic lipid layers containing a photochromic material (spiropyran) via the layer-by-layer method. The zeta potential of the lipid layers containing spiropyran was adjusted from negative to positive by photo-isomerization of spiropyran using UV illumination. A single sensor was manipulated by optical tweezers and transferred to a cell surface, thereafter adhering selectively to the cell surface under UV illumination without excess sensor adhesion. We then drove the focal point of the optical tweezers to move up and down circularly near the sensor, mimicking a vibration on the sensor or rapid injection. The surface zeta potential of the liposome layers was measured using a zeta potential analyzer. The fluorescence resonance energy transfer (FRET) method was used to observe the changes in contact area between the adhered sensor and cell membrane before and after vibration. Holographic optical tweezers (HOT) and laser confocal microscopy were used to manipulate the single sensor and to capture
\end{abstract}

(C) 2015. This manuscript version is made available under the Elsevier user license http://www.elsevier.com/open-access/userlicense/1.0/ 
fluorescent images. The results showed that the vibration applied on the sensor could push down the sensor, inducing a downward displacement. This displacement caused a corresponding deformation of the cell membrane, which increased the contact area between the sensor and the cell membrane. Without vibration, the sensor was injected into the cytoplasm in $5 \mathrm{~h}$ at an injection rate of $40 \%$. By applying the vibration stimulus, we succeeded in the rapid injection of the sensor in $30 \mathrm{~min}$ at an injection rate of $80 \%$.

Keywords: Fluorescence; Optical tweezers; Lipofection; Vibration stimulus; Optical injection

\section{Introduction}

Cellular events, including cell division, gene expression, enzyme reaction, and metabolism, are fundamentally regulated by the intracellular chemical environment (temperature, $\mathrm{pH}$, and dissolved oxygen) [1-3]. Even a very small change in temperature or $\mathrm{pH}$ can lead to different reactions within a cell. The cellular pathogenesis of diseases is always characterized by extraordinary heat production or $\mathrm{pH}$ change within a cell. It has been reported that the heat production is also greater in tumor cells than that of normal cells [4], and the intracellular $\mathrm{pH}$ of MDCK cells dropped by some 0.3-0.4 units between 3 and $5 \mathrm{~h}$ after influenza virus infection [5]. So an accurate method of measuring intracellular temperature and $\mathrm{pH}$ could thus help clarify intricate cellular processes and develop new applications in biology and medicine. Thus, the development of micro-nano sensors with high sensitivities to temperature and $\mathrm{pH}$, high spatial resolution, and effective injection technologies into single cells has become an urgent demand.

In the past, many kinds of biocompatible micro-nano sensors that can detect changes in temperature and $\mathrm{pH}$ have been developed. And in order to pass through the cell membrane, a number of advanced biological, chemical, and physical methodologies have been developed in the last three decades, including micro-nano pipette injection [6], endocytosis and lipofection 
[7], optoporation [8], and optical injection [9]. Fluorescent polymeric nanoparticles which could diffuse throughout the cell for intracellular temperature mapping had been developed by $\mathrm{K}$. Okabe et al [2]. They succeeded in the intracellular temperature imaging in living HeLa cells. A $\mathrm{pH}$-sensitive nano-sensor had been developed for intracellular $\mathrm{pH}$ sensing and tracking by $\mathrm{S}$. Chen et al [10]. Upon diffusing into cells, the sensor had been successfully applied for $\mathrm{pH}_{\mathrm{i}}$ imaging and monitoring with the use of confocal microscopy. Gold nanoparticles had been successfully injected through phospholipid membranes in less than 1 second by a laser beam as reported by Alexander S. Urban et al [11]. It relied on the strong optical forces acting on and efficient plasmonic heating of gold nanoparticles exposed to laser light for photoporation of phospholipid membranes. Intracellular delivery of gold coated liposomes encapsulating active molecules by optical injection had been introduced by Gabriel V. Orsinger et al [9]. But in these researches, either they are not targeting means and not quantitative injection of the sensors into cytoplasm or interference factors (heat, perforation, et al) are leaded to cells which will be a big stimulus or damage to the cell. These limitations will reduce the accuracy of intracellular measurement. The simultaneous measurements of temperature and $\mathrm{pH}$ within cells are also an attractive topic in life science. In order to measure local intracellular conditions, effective and rapid injection technologies with low stimulus to the target cell are in great demand.

Spiropyran is a type of organic chemical compound, known for photochromic properties that provide this molecule with the ability of being used in medical and technological areas. There have been many studies on photochromism in spiropyrans [12]. Optical tweezers, which are arguably the most versatile single-molecule manipulation technique, are widely used in single-molecule measurements, optical injection of small particles, and cell palpation. Optically driven transport into a living cell had been done successfully by S. M. Nomura et al [13]. In our previous research, we have synthetized multi fluorescent micro-sensors ( $\Phi$ 1um) which can 
respond to both temperature and $\mathrm{pH}$ change of surroundings. In order to realize selective adhesion and quantitative injection of a single sensor on cell surface, in the study of this paper, we introduced liposome layers containing photochromic material (spiropyran) on the surface of the micro-sensor. Zeta potential of the liposome layers can be switched between negative and positive by photoisomerization of spiropyran. Then we propose rapid injection of fluorescence sensors into a target cell by applying local vibration stimulus using optical tweezers. We also compare the results of rapid injection with those of injection without any stimulus on the cell membrane.

\section{Principle of optical injection using optical control of zeta potential and optical manipulation}

Figure 1 shows the principle of the rapid optical injection of a single sensor into a cell using optical tweezers. Before the injection experiment, the surface of the sensor was modified by functional liposome layers. Lipid layers have been used for encapsulation and controlled delivery of soluble drugs [14] and polymer particles [15] into cells for lipofection. Lipofection, as shown in Fig.2, is a technique used to inject biological particles into a cell by means of liposomes, which can easily merge with the cell membrane since they are both made of a phospholipid bilayer. CH.X. Bao et al had proved the lipid fusion of two membrane-coated beads and they also cited that the whole lipid fusion process involved the docking, hemifusion, and full fusion of the bilayer [16]. So the sensor encapsulated in liposome could be delivered into cytoplasm by lipofection. Furthermore, we applied vibration stimulus using optical tweezers on the liposome-encapsulated sensor to promote the membrane fusion process. In the optical tweezers, a small object is trapped by the force of radiation pressure exerted by a focused laser beam [17]. Radiation force can hold the object and translate it with high spatial 
resolution in the sub-micrometer scale. We took advantage of the optical tweezers and drove them to move up and down circularly near the sensor, which has adhered to the cell membrane. A periodic trapping force was experienced by the sensor, which was similar to a vibration on the sensor and cell membrane. G. Apodaca and the coworkers had done the research on the mechanical stretch-regulated exocytosis/endocytosis in bladder [18]. They found that the stretch on umbrella cell membrane could enhance both exocytosis and incorporation of vesicle cargo into the apical membrane. So the vibration stimulus on sensor and local cell membrane in our study could probably promote lipid fusion between liposome layers on the sensor and cell membrane and induce a rapid injection of the sensor into the cell.

\section{Materials and Methods}

\subsection{Materials}

Polystyrene (PS) microbeads with amino group-modified surfaces $(1 \mu \mathrm{m}$ in diameter, manufactured by Polysciences Inc.) were used as the sensor carriers. Two indicators (Rhodamine $\mathrm{B}$ and FITC) were used to stain the PS microbeads. 1,2-dipalmitoyl-sn-glycero-3-phosphatidylcholine (DPPC) and 1,2-dipalmitoyl-sn-glycero-3phosphatidylglycerol (DPPG) were used as liposomes (manufactured by NOF CORPORATION). DPPC is a cationic liposome and DPPG is an anionic liposome. 1',3',3'-Trimethyl-6-nitrospiro [1(2H)-benzopyran-2,2'-indoline] (SP) (Tokyo Chemical Industry Co., Ltd.) was used as the photochromic material that was mixed with the liposomes.

\subsection{Cell culture}

Madin-Darby canine kidney (MDCK) cells were used for experiments. Before injection experiment, the cells are cultured in glass base dish ( $\Phi 3 \mathrm{~cm}$, ASAHI GLASS CO. LTD. JAPAN) in the incubator with a condition of $37^{\circ} \mathrm{C}$ bubbled with $95 \%$ air, $5 \% \mathrm{CO}_{2}$ gas. Minimum 
essential medium eagle is used as cell medium containing $10 \%$ fetal bovine serum (FBS). For injection experiment, the glass dish containing cells is moved to experimental chamber in the experimental system. The temperature in the chamber is controlled to $37^{\circ} \mathrm{C}$ with a gas injection of $\mathrm{CO}_{2}$. The condition in the chamber is suitable for cell culture which makes it possible to be used for experiment.

\subsection{Preparation of lipid vesicles}

In order to encapsulate the sensor using liposome layers, we first prepared lipid vesicles as follows. The lipids and spiropyran (SP) were first mixed in test tubes with chloroform solutions $\left(\mathrm{C}_{\text {lipids }}=0.2 \mathrm{mM}, \mathrm{C}_{\mathrm{SP}}=0.04 \mathrm{mM}\right)$. To form dry lipid films, the mixtures were dried in an evaporator under a water-heating temperature of $40{ }^{\circ} \mathrm{C}$. The thoroughly dried lipid films were hydrated in deionized (DI) water to form multi-lamellar vesicles (MLVs) and then transformed into small unilamellar vesicles (SUVs) by sonication (60 min, $50 \mathrm{~W}, 0.4 \mathrm{~s}$ pulse). The resulting solution was strained through a filter with a pore size of $0.4 \mu \mathrm{m}$.

\subsection{Fabrication of multi-fluorescence sensor and encapsulation by functional liposomes}

We previously fabricated multi-fluorescent sensors that could respond to both temperature and $\mathrm{pH}$ [19]. Rhodamine B (ex. $561 \mathrm{~nm}$ ), which could respond to changes in temperature, was embedded inside the PS beads. FITC (ex. $488 \mathrm{~nm}$ ), which could respond to changes in both $\mathrm{pH}$ and temperature, was immobilized on the surface of the beads. The temperature and $\mathrm{pH}$ sensitivities of the sensor has been studied and discussed in previous work [19].

Figure 3 shows the encapsulation process of the individual sensor in lipid layers fabricated via the layer-by-layer method. Bilayers were assembled on the beads via the same vesicle adsorbing/spreading process as reported by J.T. Groves and coworkers [20]. The fabricated fluorescent sensors were suspended in DI water with a final concentration of $0.5 \mathrm{wt} \%$. The 
sensor (100 $\mu \mathrm{L}$ in volume) and the SUV solution (900 $\mu \mathrm{L}$ in volume) were combined in a small centrifuge tube, and the mixture was subjected to pulse vortex. Excess vesicles were removed by rinsing several times with DI water. After removing all of the DI water, $1 \mathrm{~mL}$ of $\mathrm{CaCl}_{2}$ solution $(100 \mathrm{mM})$ was added to the same tube, as calcium ions could induce the fusion of SUVs, and a solid supported membrane was formed on the beads. A double-lamellar layer of positively charged DPPC was first prepared on the surface of the sensor, and then negatively charged DPPG was coated on the outside of DPPC, as shown in Fig. 3.

\subsection{Surface charge control of the sensor by photoreaction of spiropyran}

Spiropyran (SP), which is a type of photochromic material [21], was included in the lipid layers. While SP was isomerized into a colored zwitterionic structure (merocyanine type) by UV illumination, the isomerized SP was isomerized back into a colorless non-ionic structure (SP type) by visible light illumination, as shown in Fig. 4 (a). This structural change facilitated the optical control of zeta potential. The zeta potential of DPPG, which was located at the surface of the liposome, was negative. The zeta potential of the UV-irradiated liposome was switched from negative to positive as shown in Fig. 4 (b). Most animal cells, for example Madin-Darby canine kidney (MDCK) cells [22], possess negative surface potential. Therefore, we could regulate the adhesion of a single sensor to the cell surface via the optical control of zeta potential. In our study, we measured the zeta potential of PS beads, amino-PS beads, DPPC liposomes with SP, and DPPG liposomes with SP using a zeta potential analyzer at an illumination wavelength of $600 \mathrm{~nm}$ (Zetasizer Nano ZS, Malvern).

\subsection{Mechanism of fluorescence resonance energy transfer (FRET)}

Fluorescence resonance energy transfer (FRET) is the physical phenomenon of the 
radiationless transfer of energy between two light-sensitive molecules [23]. Fluorescence energy transfer occurs via the overlapping of the spectrum of a fluorophore (donor) with the absorption spectrum of a molecule (acceptor), as shown in Fig. 5. The excited donor fluorophore emits a virtual photon that is instantly absorbed by the acceptor molecule. According to the theory of fluorescence resonance energy transfer [23], the transfer energy is very sensitive to the distance between the donor and the acceptor, as described in Equation 1.

$$
E=1-\frac{F}{F_{O}}=\frac{R_{O}^{6}}{R_{O}^{6}+r_{O}^{6}}
$$

$F$ and $F_{0}$ are the fluorescence intensities of the donor in the presence and absence of a quencher, respectively, $r_{0}$ is the distance between the acceptor and the donor, and $R_{0}$ is the critical distance when the transfer efficiency is $50 \%$. The distance between the donor and the acceptor is typically in the range of 1-10 $\mathrm{nm}$. In our research, the FRET method was used to observe the contact area between the adhered sensor and the cell membrane. The cell membrane could be excited by the fluorescent emission of the sensor, which has adhered to the cell membrane.

\section{Experiments}

\subsection{Experimental setup}

We constructed an optical system based on a commercial inverted microscope (IX71, Olympus), consisting of holographic optical tweezers (HOT) and a laser confocal scanning system as shown in Fig. 6. The HOT system has been designed and reported in our previous work [24]. An infrared laser (YLM-10-1064, IPG PHOTONICS, maximum power: $10 \mathrm{~W}$, wavelength: $1064 \mathrm{~nm}$ ) was used as a light source. The laser beam was modulated with a spatial light modulator (SLM: Hamamatsu Photonics X10468-03, frame rate: $60 \mathrm{~Hz}$ ) [25], which 
displayed a Kinoform hologram. Then the laser beam was transferred to the back focal plane of the microscope objective lens (Olympus, UPLSAPO 100XO, 100×, numerical aperture: 1.40 , working distance: $130 \mu \mathrm{m}$. The laser spot was generated on the focal plane of the objective lens and the target object was trapped at the laser spot. The $\mathrm{Z}$ piezo stage was fixed to the objective lens so that the movements of the laser spot and the objective lens could be controlled by the $\mathrm{Z}$ piezo stage. The manipulation of the trap point of one bead to vibrate near a certain region of the cell membrane was applied by the HOT system, and the manipulation was observed with an analog CCD camera (WAT-221S, Watec. Co. Ltd.). Three-dimensional fluorescent images were taken using a laser confocal scanning system (CSU-X1, Yokogawa. Co. Ltd.) with two excitation lasers at $488 \mathrm{~nm}$ and $561 \mathrm{~nm}$ and an electron-multiplying charge-coupled device (EM-CCD) (iXon, Andor). A Z stage for the objective lens (P-E662, Physik Instrumente GmbH Co.) was used to acquire 3D slice images. A fluorescence system equipped with a mercury lamp was also used to induce photo-isomerization by UV illumination.

4.2 Selective adhesion of liposomes to the cell membrane using optical manipulation

The sensor-encapsulated lipid layers were trapped by the optical tweezers and placed in contact with the cell surface under visible light (VIS). The sensor was then illuminated by ultraviolet (UV) light for $1 \mathrm{~min}$, and the zeta potential of the liposomes could be switched from negative to positive via the photo-isomerization of SP. In this experiment, the UV light power was $3.5 \mathrm{~mW} / \mathrm{cm}^{2}$ and the VIS power was $5.4 \mathrm{~mW} / \mathrm{cm}^{2}$. During the UV illumination, the positively charged lipid was able to adhere to the cell membrane.

4.3 Vertical vibration stimulus on the adhered sensor using optical tweezers for rapid injection As shown in Fig. 7 (a), after two sensors adhered to the same cell surface, we applied optical 
tweezers on one of the sensors to move it up and down circularly in sine mode below the sensor. In our experimental system, the laser spot of the optical tweezers was on the same plane as the focus plane of the objective lens, which was fixed to a $\mathrm{Z}$ piezo stage, so that the displacement and frequency of the moving optical tweezers could be controlled by the input voltage of the $\mathrm{Z}$ piezo stage. In our experiment, the frequency of the moving focal point of the laser was $1 \mathrm{~Hz}$ and the maximum displacement $(\Delta \mathrm{Z})$ was $4 \mu \mathrm{m}$. The displacement curve of the optical tweezers in sine mode is shown in Fig. 7 (b). The cyclic vertical movement of the focal point of the laser provided a persistent vibration stimulus on the sensor. In the vibration experiment, the peak position of the focal point of the laser was the same as the position of the adhered sensor. Therefore, the trapping force of the sensor was downward on the cell membrane when the focal point moved to a position below the sensor. After the application of the vibration stimulus, a laser confocal microscope was used to take $Z$ slice images with a scanning distance of $10 \mu \mathrm{m}$. The exact position of the sensor before and after the application of the vibration stimulus could be observed in the 3D fluorescent images.

4.4 Observation of the contact area between the adhered sensor and the cell membrane by FRET

We used the FRET method to detect the change in contact area between the adhered sensor and the cell membrane after vibration. The microbead ( $\Phi 5 \mu \mathrm{m})$ was encapsulated by liposomes, which was stained by quantum dots (Lumidot ${ }^{\mathrm{TM}} \mathrm{CdSe} / \mathrm{ZnS} 510$, ex. $366 \mathrm{~nm}$, em. $510 \mathrm{~nm}$ ) as the donor, as shown in Fig. 8. The cell membrane was stained by CellMask ${ }^{\mathrm{TM}}$ as the acceptor. The overlap of the absorption spectrum of CellMask ${ }^{\mathrm{TM}}$ with the fluorescence emission spectrum of Lumidot $^{\mathrm{TM}} 510$ is shown in Fig. 9 (a). Figure 10 shows our schematic design of the FRET experiment between a single sensor and the cell membrane. The adhered sensor, which was 
stained by Lumidot ${ }^{\mathrm{TM}} 510$, could be excited by UV illumination. The cell membrane could absorb the fluorescence emission from only one sensor that adhered to it, in turn emitting fluorescence at a longer wavelength. Therefore, the emission from the cell membrane, which was excited by FRET, should be very weak. In order to successfully observe the emission by FRET, we used microbeads with a larger size $(\Phi 5 \mu \mathrm{m})$ than those used in the injection experiment $\left(\begin{array}{lll}\Phi & 1 & \mu \mathrm{m}\end{array}\right)$. We applied the same vibration stimulus on the sensor in the FRET experiment, the experimental setup for which is shown in Fig. 9 (b). UV was illuminated by a mercury lamp, and an EM-CCD equipped with a barrier filter $(590 \mathrm{~nm})$ was used to observe the fluorescence emission from the cell membrane excited by FRET.

\section{Results}

5.1 Control of zeta potential of liposomes containing photochromic material

We measured the zeta potential of PS beads, amino-PS beads, DPPC liposomes with SP, and DPPG liposomes with SP using a zeta potential analyzer under illumination at $600 \mathrm{~nm}$ (Zetasizer Nano ZS, Malvern), and the results are shown in Table I. In table I, the measured zeta potential of DPPC is positive while that of DPPG is negative. We also examined the amount of surface charge in each material by evaluating the duration of the particle passage (the time it took for a particle to go through a pore) using a nanoparticle analysis system (qNANO, iZON). The duration increases with the zeta potential in this equipment. Figure 11 shows the calibration curve of zeta potential and the duration of passage. From this result, the relationship between the zeta potential and the duration of passage is represented in Equation 2.

$$
V_{z}=30.6 \cdot D-156
$$

$V_{z}$ is the zeta potential $[\mathrm{mV}]$ and $\mathrm{D}$ is the time $[\mathrm{ms}]$ it took the particles to pass through the pore during the particle charge analysis. In our experiment, DPPG with SP was located at the surface 
of the sensor. The measured duration of passage of the DPPG liposome under UV illumination was $5.67 \mathrm{~ms}$. From Equation 2, the zeta potential of the UV-illuminated DPPG liposome was calculated to be $17.4 \mathrm{mV}$, suggesting that we have succeeded in switching the zeta potential of the liposome-containing sensor from negative (-73.2) to positive (17.4). Actually, the photoisomerization of spiropyran is reversible. After UV was removed, the zeta potential of the liposome-containing sensor was change to negative again.

\subsection{Selective adhesion of liposome sensor to cell membrane}

We succeeded in the adhesion of a single sensor to the cell membrane by photo-isomerization, as shown in Fig. 12. A single sensor could be trapped and manipulated by optical tweezers and transferred to the cell surface (Fig. 12 (a) and (b)). The sensor has been encapsulated by liposome layers containing the photochromic material, spiropyran. Then the sensor was exposed to UV illumination for $1 \mathrm{~min}$, and adhered to the cell membrane based on the change in surface charge induced by photo-isomerization (Fig. 12 (c) and (d)). When the focal point of the laser was removed from the sensor, the sensor still adhered to the cell surface. Based on our experiment results, without UV illumination, after the sensor was transferred to cell membrane, the sensor escaped from cell surface after removing the laser point.

\subsection{Sensor injection by lipofection without vibration stimulus}

Before the injection experiment, the cell membrane of MDCK cells was stained by CellMask $^{\mathrm{TM}}$, which shows red fluorescence at ex. $561 \mathrm{~nm}$ as seen in Fig. 13. Figure 13 (a) shows the fluorescence emission spectra of CellMask ${ }^{\mathrm{TM}}$ Orange plasma membrane stains with an excitation peak at $550 \mathrm{~nm}$. Figure 13 (b) shows a 3D fluorescent image of the cell membrane taken by $\mathrm{Z}$ slicing with steps of $0.2 \mu \mathrm{m}$ and a scanning distance of $10 \mu \mathrm{m}$. In order to investigate 
the different effects of injection with and without vibration stimulus, before applying the vibration stimulus on the sensor, we detected the injection results of lipofection without any stimulus on the sensor, which are shown in Fig. 14. In the 3D fluorescent image in Fig. 14 (a), we observed the position of the sensor, which adhered to the surface of a cell. Lipofection is based on membrane fusion between the cell membrane and the liposome layers of the sensor. The result in Fig. 14 (b) shows that within $3 \mathrm{~h}$ after adhesion, the sensor was embedded in the cell membrane, indicating that membrane fusion occurred during lipofection. It took almost $5 \mathrm{~h}$ for the sensor $(\Phi 1 \mu \mathrm{m})$ to be completely injected into the cell, as shown in Fig. 14 (b). Of the ten sensors that adhered to different cell surfaces, only four were injected into the cells in $5 \mathrm{~h}$. Therefore, the injection time of lipofection was $5 \mathrm{~h}$ at an injection rate of $40 \%$.

5.4 Change in contact area between the adhered sensor and the cell membrane after vibration The sensor was encapsulated by lipid layers that were stained by quantum dots (Lumidot ${ }^{\mathrm{TM}}$ CdSe-ZnS 510, ex. $366 \mathrm{~nm}$, em. $510 \mathrm{~nm}$ ) and a fluorescent image of the sensor is shown in Fig. 15. Figure 15 (a) shows the typical absorption and photoluminescence spectra of Lumidot ${ }^{\mathrm{TM}} 510$. The quantum dots can be excited by UV light $(366 \mathrm{~nm})$ and shows fluorescent emission in the region of $450 \mathrm{~nm}$ to $550 \mathrm{~nm}$, as shown in Fig. 15 (c). They show no emission at wavelengths that are longer than $590 \mathrm{~nm}$, as shown in Fig. 15 (d).

The FRET results are shown in Fig. 16, where we observed three sensors in Fig. 16 (a). Sensor 1 and sensor 2 adhered to the cell membrane and sensor 1 was later vibrated by optical tweezers. Sensor 3 adhered to the glass dish. In the FRET experiment, UV illumination was used to excite the sensor to emit fluorescence, which could be absorbed by the cell membrane. EM-CCD equipped with a barrier filter $(590 \mathrm{~nm})$ was used to observe the emission fluorescence of the cell membrane. Figure 16 (b) shows the fluorescent image of the cell membrane excited 
by FRET before the vibration experiment. The red fluorescence in Fig. 16 (b) was expressed by the cell membrane, which was excited by the sensor that adhered to it. $d_{1}$ and $d_{2}$ show the diameters of the fluorescent membrane in the positions of Sensor 1 and Sensor 2, respectively. $\mathrm{d}_{1}$ and $\mathrm{d}_{2}$ were almost same $\left(\mathrm{d}_{1}=1.76 \mu \mathrm{m}, \mathrm{d}_{2}=1.83 \mu \mathrm{m}\right)$ before vibration was applied on Sensor 1. $d_{1}$ increased to $3.64 \mu \mathrm{m}$ while a very small change occurred for $d_{2}(1.97 \mu \mathrm{m})$ after vibration was applied on Sensor 1 for 20 min, as shown in Fig. 16 (c). These observations suggest that the vibration on Sensor 1, which was applied by the cyclic vertical movement of the optical tweezers, could push down the sensor and induce a downward displacement. As shown in the upper diagram in Fig. 16 (c), the downward displacement of the sensor induced a corresponding deformation of the cell membrane, which increased the contact area between the sensor and the cell membrane, resulting in the increase in $\mathrm{d}_{1}$.

5.5 Optical results of rapid injection by optical tweezers with local vibration stimulus

Figure 17 shows the results of rapid sensor injection by applying a vibration stimulus using optical tweezers. Figures 17 (a)-(c) are bright-field images and Fig. 17 (d)-(f) are 3D fluorescent images taken in multi-channels of red and green. In our experimental system, the focal point of the laser moved with the $\mathrm{Z}$ piezo stage in sine mode with a frequency of $1 \mathrm{~Hz}$ and an amplitude of $4 \mu \mathrm{m}$. The displacement and frequency of the focal point could be controlled by the input voltage of the $\mathrm{Z}$ piezo stage. Two sensors adhered to the cell surface before vibration, as shown in Fig. 17 (a) and (d). The cell membrane shows fluorescence in the red channel (ex. $561 \mathrm{~nm}, \mathrm{em} .590 \mathrm{~nm}$ ) and the sensor shows fluorescence in the green channel (ex. $488 \mathrm{~nm}$, em. $510 \mathrm{~nm}$ ). After applying vibration stimulus for $20 \mathrm{~min}$ on Sensor 1 and also $10 \mathrm{~min}$ after vibration, the bright-field images in Fig. 17 (b) and (c) show an apparent difference in the vertical position of the two sensors, suggesting that a displacement between Sensor 1 and 
Sensor 2 was induced by the vibration. In the 3D fluorescent image in Fig. 17 (e), Sensor 1 has been pushed down by the optical tweezers compared with Sensor 2. The displacement of Sensor 1 induced a corresponding deformation of the cell membrane, but the sensor was still on the cell surface. Figure 17 (f) shows that 10 min after the vibration was applied, the sensor was totally injected into the cytoplasm. In the 3D fluorescent image of Fig. 17 (f), it is clear that Sensor 1 was below the cell membrane while Sensor 2 was still on the cell surface. In fact, we observed ten sensors that adhered to different cell surfaces, and eight of them were injected into the cytoplasm in $30 \mathrm{~min}$. Thus, in comparison with the lipofection method, which had an injection time of $5 \mathrm{~h}$, the presence of local vibration stimulus on the sensors shortened the injection time to $30 \mathrm{~min}$. Additionally, the injection rate was also increased to $80 \%$ compared with that of lipofection (40\%).

\section{Discussions}

In our research, we succeeded in the selective adhesion and rapid injection of a single micro-sensor into a target cell. Comparing with the nano-sensors which have been studied by many researchers, the injection of micro-sensors into target cells has several merits. For the polymeric nanoparticles, it can always pass through the cell membrane by diffusion and endocytosis after incubating the cells with the nanoparticles $[2,26]$. It not only takes several hours for the delivery of the nanoparticles into cytoplasm but also non - quantitative. For the nanoparticles modified by some metal particle, it is commonly delivered into cell by optical methods $[9,11]$. The optical injection of metallic nanoparticles can provide a very short injection time (as short as 1 several seconds), however it also leads to localized heating-up on cell membrane. The heating-up on cell membrane is a big interference especially during the temperature measurement of the cell. Using our proposed method, we can control the number of 
sensors introduced to cells which can be as small as a single sensor. The very low stimulus to the cell during the injection is thinkable based on the small injection number of sensors and the very weak force of optical tweezers. And no heat is produced by optical operation since our sensor is based on polymeric material. What we want to mention here is that our established optical system is consisting a holographic optical tweezers (HOT) which makes it possible to manipulate several micro-sensors as same time. Then the micro-sensors can be transferred to different regions on cell membrane and then be injected into the cell. As cells are noisy systems, injection of several micro-sensors into different places in cytoplasm will be convincing. The number of injected sensors for detection is really small, so the sensor in micro scale will be easy for fluorescence detection after injected in cytoplasm.

G. Apodaca et.al cited on the role of mechanical forces on endocytosis [18]. They reported that mechanical stretch stimulates apical endocytosis in bladder umbrella cells. In our study, the mechanical stimulus was driven by optical tweezers which was weak a lot comparing with stretch. And MDCK cells that we used are totally different with bladder umbrella cells. The membrane area of MDCK cell cannot expand as large scale as that of bladder umbrella cells. So in our case, the mechanical force-induced endocytosis is only to a limited extent. The fluorescent sensor that we used was encapsulated in liposome layers which had been used widely for lipofection delivery of biological particles [27, 28]. The liposome layers can easily merge with the cell membrane since they are both made of a phospholipid bilayer. In our previous work, nano-sensors encapsulated in liposome layers have been transfected into cell nucleus by lipofection [15]. Actually not only stretch-induced endocytosis had been noted by G. Apodaca et.al, it had been also demonstrated that mechanical stretch on cell membrane also resulted in the delivery and incorporation of vesicle cargo into the apical membrane [18]. Our research results are coincident with that mechanism. The mechanical force driven by optical 
tweezers increased the liposome merging with cell membrane. So the injection of sensor into cell by lipofection was enhanced by mechanical force.

In the fluorescent image of the sensor after injection into cytoplasm, the sensor also shows good fluorescence which makes it possible to track and observe the sensor. It had been proved that our fabricated fluorescent sensor could endure low $\mathrm{pH}(\mathrm{pH}=4)$, and can also respond to low $\mathrm{pH}$ repeatedly. So the sensor will be an effective mean for intracellular measurement even in acidic organs. The observation of sensor location and measurements in cytoplasm after sensor injection will be urgent works in future. The sensor is expected for its applications in intracellular measurement in different organs.

\section{Conclusions}

A novel multi-fluorescent sensor has been synthesized based on amino-polystyrene microbeads using Rhodamine B and FITC. It was able to respond to changes in both temperature and $\mathrm{pH}$. A single sensor was successfully manipulated by optical tweezers and adhered selectively to the cell surface via the optical control of the zeta potential of spiropyran. Rapid sensor injection was achieved with an injection time of approximately $30 \mathrm{~min}$ by applying cyclic vibration stimulus using optical tweezers. With the vibration method, the injection rate was also increased to $80 \%$ compared with that observed in the lipofection method. This study is fundamentally helpful in the delivery of micro-nano particles used in biomedical applications, such as delivery of drugs, genes, or contrast agents for biomedical imaging and therapy. At the moment, we are seeking to extend this injection method to other cell lines. Since the membranes of most cell types are constructed from similar lipids, we expect that the rapid injection method will work for most cell lines. This technique also makes it possible to measure intracellular $\mathrm{pH}$ and temperature, which will be a great contribution to cell biology. We believe 
that our work will support breakthroughs in the development of new medicine and drug therapy in cancers and viral diseases.

\section{Acknowledgements}

This work has been supported by Core Research for Evolutional Science and Technology (CREST) of JST.

\section{References}

[1] D.A. Warner, R. Shine, The adaptive significance of temperature-dependent sex determination in a reptile, Nature 451 (2008) 566-569.

[2] K. Okabe, N. Inada, C. Gota, Y. Harada, T. Funatsu, S. Uchiyama, Intracellular temperature mapping with a fluorescent polymeric thermometer and fluorescence lifetime imaging microscopy, Nat. Commun. 3 (2012) No. 705.

[3] S. Humez, M. Monet, F. van Coppenolle, P. Delcourt, N. Prevarskaya, The role of intracellular pH in cell growth arrest induced by ATP, Am. J. Physiol.-Cell Ph. 287 (2004) $1733-1746$.

[4] M. Monti, L. Brandt, J. Ikomi-Kumm, H. Olsson, Heat production rate in blood lymphocytes as a prognostic factor in non-Hodgkin's lymphoma, Eur. J. Haematol. 45 (1990) 250-254.

[5] F. Ciampor, C.A. Thompson, S. Grambas, A.J. Hay, Regulation of $\mathrm{pH}$ by the M2 protein of influenza A viruses, Virus Res. 22 (1992) 247-258.

[6] H. Maruyama, N. Inoue, T. Masuda, F. Arai, Selective injection and laser manipulation of nanotool inside a specific cell using optical $\mathrm{pH}$ regulation and optical tweezers, in: IEEE International Conference on Robotics and Automation (ICRA), Shanghai, 2011, pp. 2674-2679. 
[7] D. Delgado, A. del Pozo-Rodríguez, M.Á. Solinís, A. Rodríguez-Gascón, Understanding the mechanism of protamine in solid lipid nanoparticle-based lipofection: The importance of the entry pathway, Eur. J. Pharm. Biopharm. 79 (2011) 495-502.

[8] L. Gu, S.K. Mohanty, Targeted microinjection into cells and retina using optoporation, J. Biomed. Opt. 16 (2011) 128003.

[9] G.V. Orsinger, J.D. Williams, M. Romanowski, Focal activation of cells by plasmon resonance assisted optical injection of signaling molecules, ACS Nano 8 (2014) 6151-6162.

[10] S. Chen, Y. Hong, Y. Liu, J. Liu et al, Full-range intracellular $\mathrm{pH}$ sensing by an aggregation-induced emission-active two-channel ratiometric fluorogen, J. Am. Chem. Soc. 135 (2013) 4926-4929.

[11] A. S. Urban, T. Pfeiffer, M. Fedoruk, A. A. Lutich, J. Feldmann, Single-step injection of gold nanoparticles through phospholipid membranes, ACS Nano 5 (2011) 3585-3590.

[12] B. S. Lukyanov, M. B. Lukyanova, Spiropyrans: synthesis, properties, and application, Chem. Heterocycl. Compd. 41 (2005) 281-311.

[13] K. Kubo, M. Ichikawa, K. Yoshikawa, Y. Koyama, T. Niidome, T. Yamaoka, S. M. Nomura, Appl. Phys. Lett. 83 (2003) 2468-2470.

[14] T.S. Troutman, J.K. Barton, M. Romanowski, Biodegradable plasmon resonant nanoshells, Adv. Mater. 20 (2008) 2604-2608.

[15] T. Masuda, H. Maruyama, A. Honda, F. Arai, Multi-layered liposome containing nanosensor for transfecting into a cell nucleus, in: IEEE 14th international conference on nanotechnology (IEEE-NANO), Toronto, 2013, pp. 162-165.

[16] CH.X. Bao, G. Pähler, B. Geil, A. Janshoff, Optical fusion assay based on membrane-coated spheres in a 2D assembly, J. Am. Chem. Soc. 135 (2013) 12176-12179. [17] K.C. Neuman, S.M. Block, Optical trapping, Rev. Sci. Instrum. 75 (2004) 2787-2809. 
[18] S.T. Truschel, E. Wang, W.G. Ruiz, S.M. Leung, R. Rojas, J. Lavelle, M. Zeidel, D. Stoffer, G. Apodaca, Stretch-regulated Exocytosis/Endocytosis in Bladder Umbrella Cells, Mol. Biol. Cell 13 (2002) 830-846.

[19] H.J. Liu, H. Maruyama, T. Masuda, A. Honda, F. Arai, Multi-fluorescent micro-sensor for accurate measurement of $\mathrm{pH}$ and temperature variations in micro-environments, Sens. Actuators B Chem. 203 (2014) 54-62.

[20] E.M. Winter, J.T. Groves, Surface Binding Affinity Measurements from Order Transitions of Lipid Membrane-Coated Colloidal Particles, Anal. Chem. 78 (2006) 174-180.

[21] R. Blonder, E. Katz, I. Willner, V. Wray, A.F. Bückmann, Application of a nitrospiropyran-FAD-reconstituted glucose oxidase and charged electron mediators as optobioelectronic assemblies for the amperometric transduction of recorded optical signals: control of the "on"-"off" direction of the photoswitch, J. Am. Chem. Soc. 119 (1997) $11747-11757$.

[22] M. Paulmichl, F. Friedrich, E. W611, H. Weiss, and F. Lang, Effects of serotonin on electrical properties of Madin-Darby canine kidney cells, Pflugers Arch. 411 (1988) 394-400.

[23] V. Helms, Principles of Computational Cell Biology, Weinheim: Wiley-VCH, Germany, 2008.

[24] K. Onda, F. Arai, Multi-beam bilateral teleoperation of holographic optical tweezers, Opt. Express 20 (2012) 3633-3641.

[25] N. Mukohzaka, N. Yoshida, H. Toyoda, Y. Kobayashi, T. Hara, Diffraction efficiency analysis of a parallel-aligned nematic-liquid-crystal spatial light modulator, Appl. Opt. 33 (1994) 2804-2811.

[26] P. G. Coupland, S. J. Briddon, J. W. Aylott, Using fluorescent pH-sensitive nanosensors to report their intracellular location after Tat-mediated delivery, Integr. Biol.1 (2009) 318-323. 
[27] P. L. Felgner, T. R. Gadek, M. Holm, R. Roman, H. W. Chan, M. Wenz, J. P. Northrop, G. M. Ringold, and M. Danielsen, Lipofection: a highly efficient, lipid-mediated DNA-transfection procedure, Proc. Natl. Acad. Sci. USA 84 (1987) 7413-7417.

[28] J. P. Yang, L. Huang, Overcoming the inhibitory effect of serum on lipofection by increasing the charge ratio of cationic liposome to DNA, Gene Ther. 4 (1997), 950-960. 


\begin{tabular}{lccccc}
\hline & \multicolumn{4}{c}{ Material and condition } \\
\cline { 2 - 6 } & PS bead & Amino-P & DPPC & DPPG \\
& & S bead & (VIS) & (VIS) \\
\hline Zeta potential & $\mathbf{m V}$ & -54 & -47.4 & 10.4 & -73.2 \\
\hline
\end{tabular}

Table I Zeta potential of each material measured by zeta potential analyzer 


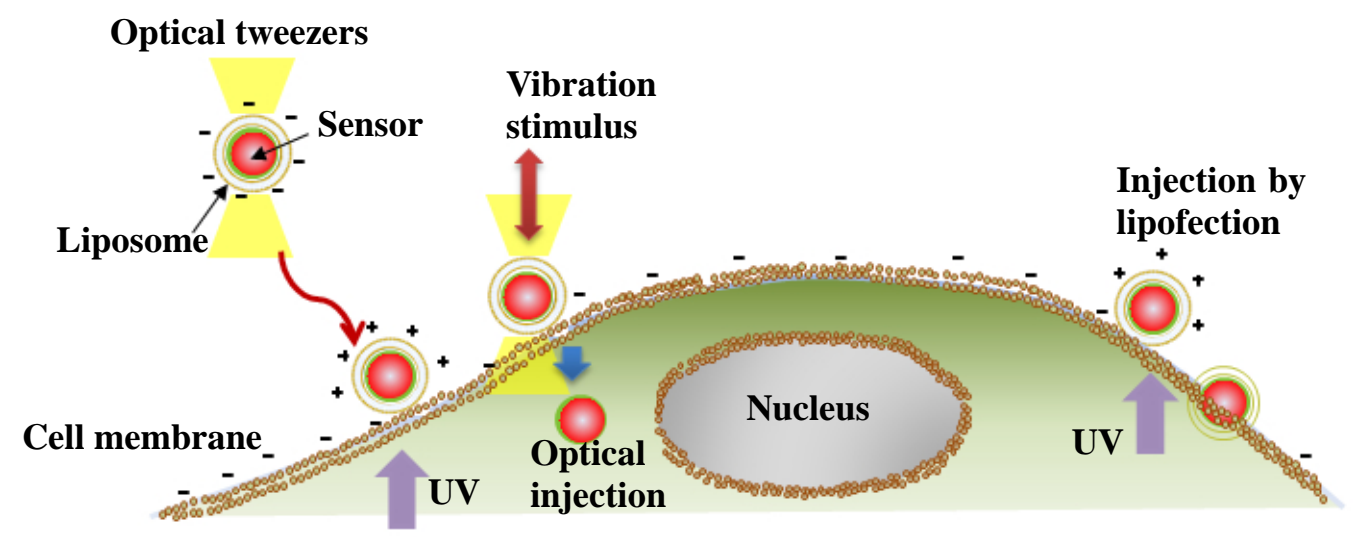

Fig. 1 A schematic diagram of selective adhesion and rapid injection of a fluorescent sensor into a target cell using local mechanical stimulus applied by optical tweezers.

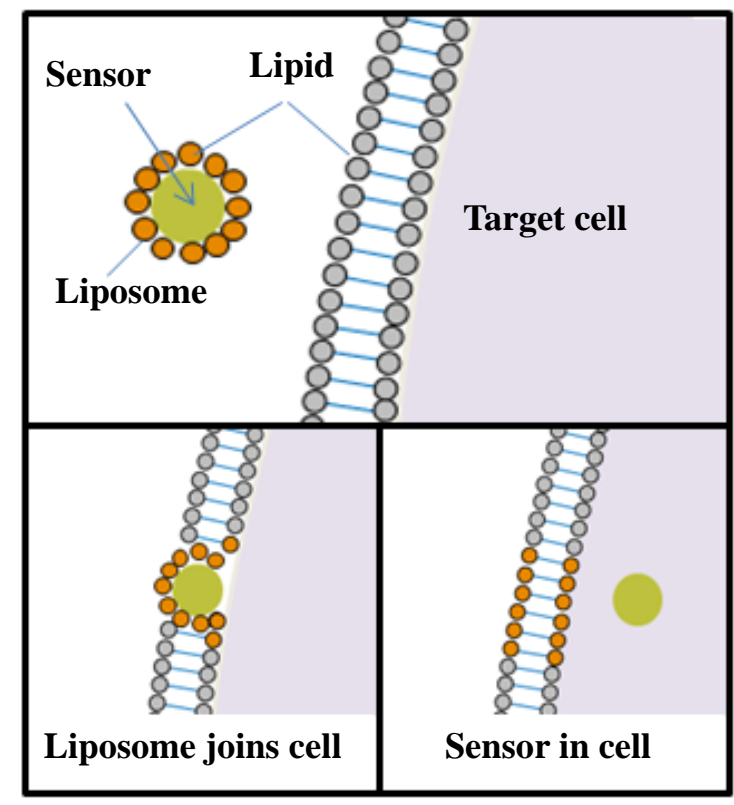

Fig. 2 diagrammatic sketch for lipofectionmechanism 


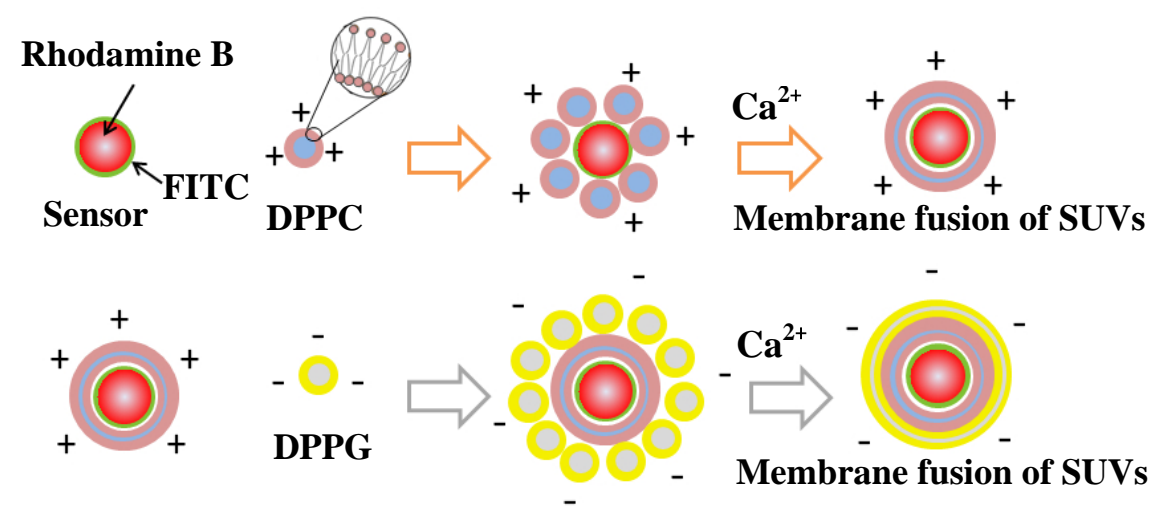

Fig. 3 Encapsulation of the sensor into the liposomes.

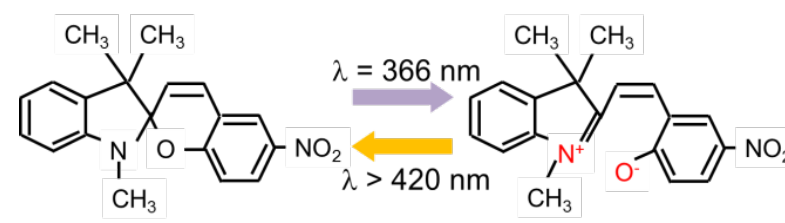

(a)

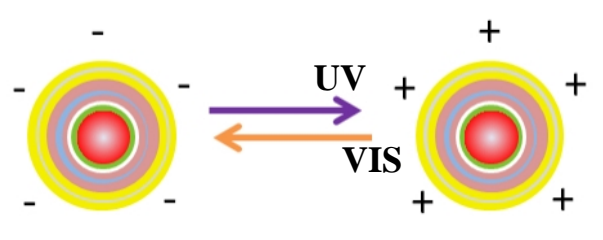

(b)

Fig. 4 Optical control of surface charge of liposomes by photo-isomerization action. (a) Isomerization of spiropyran by UV/VIS illumination. (b) Control of the surface charge of liposomes by UV/VIS illumination.

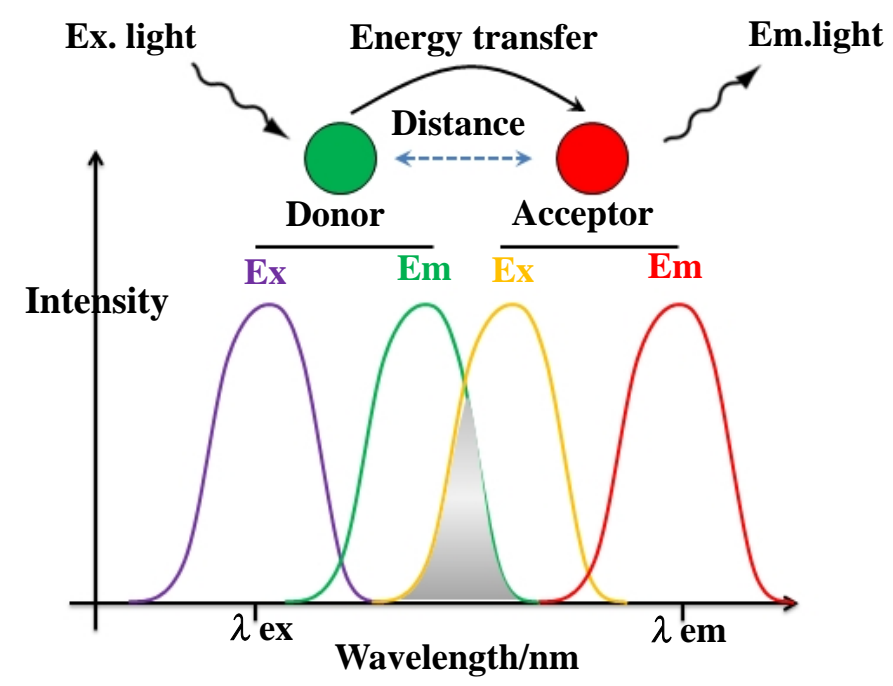

Fig. 5 The mechanism of fluorescence resonance energy transfer (FRET). 


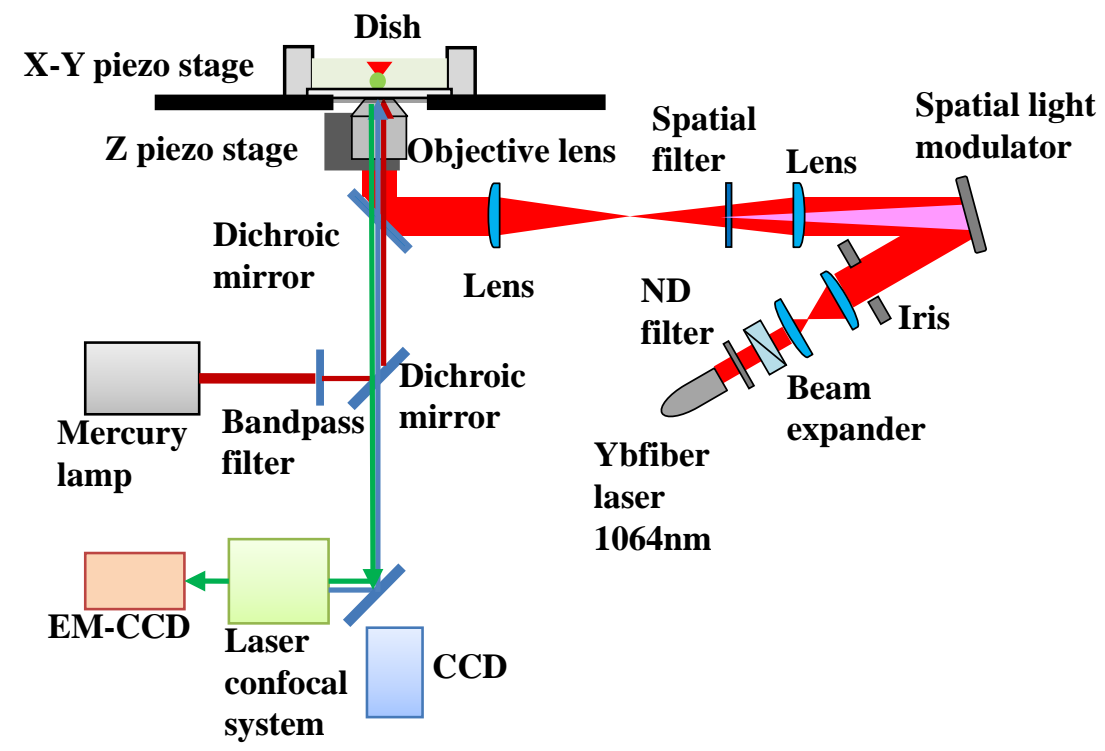

Fig.6 Setup of optical tweezers system and inverted confocal microscopy system.

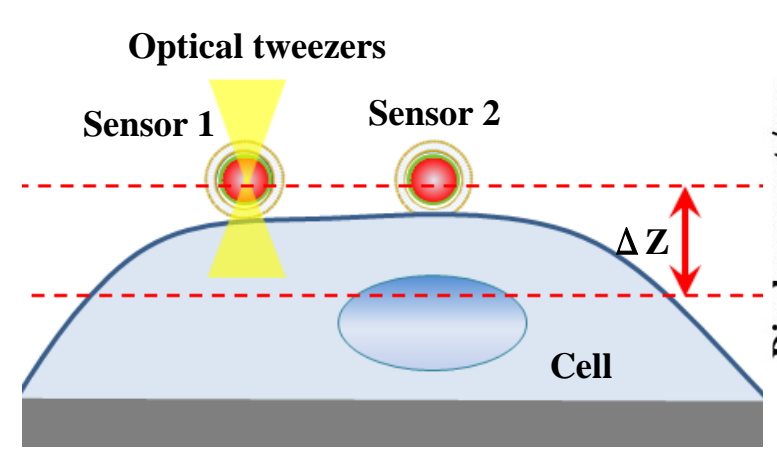

(a)

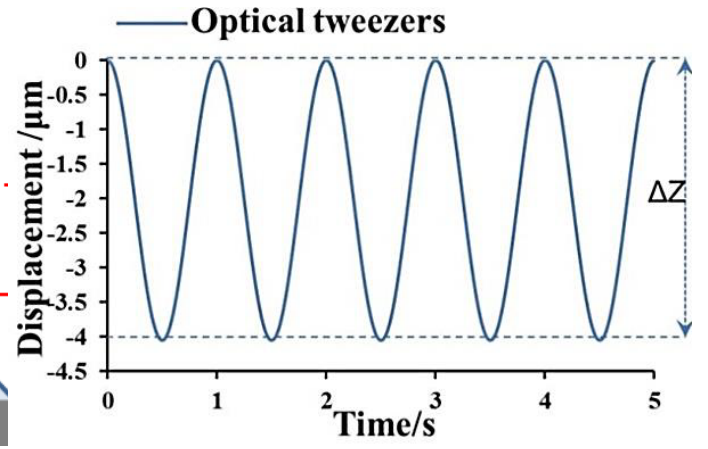

(b)

Fig. 7Vertical vibration stimulus on the adhered rapid injection sensor. (a) Schematic diagram of the local injection of a sensor with vibration stimulus applied by optical tweezers. (b) The displacement curve of the laserfocal point in 5 cycles in sine mode with a frequency of $1 \mathrm{~Hz}$ and $\Delta \mathrm{Z}$ of $4 \mu \mathrm{m}$. 


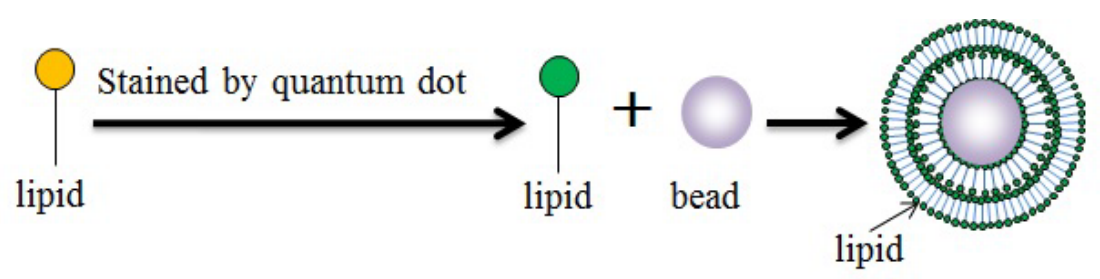

Fig. 8 The fabrication of a fluorescent microbeadencapsulated by a lipid membrane, stained by quantum dots (Lumidot ${ }^{\mathrm{TM}}$ CdSe/ZnS 510, ex. 366 nm, em. 510 nm).

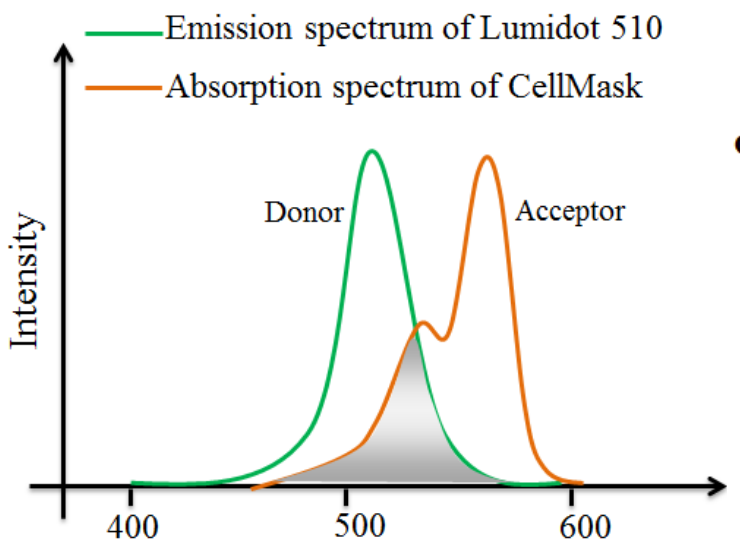

(a)

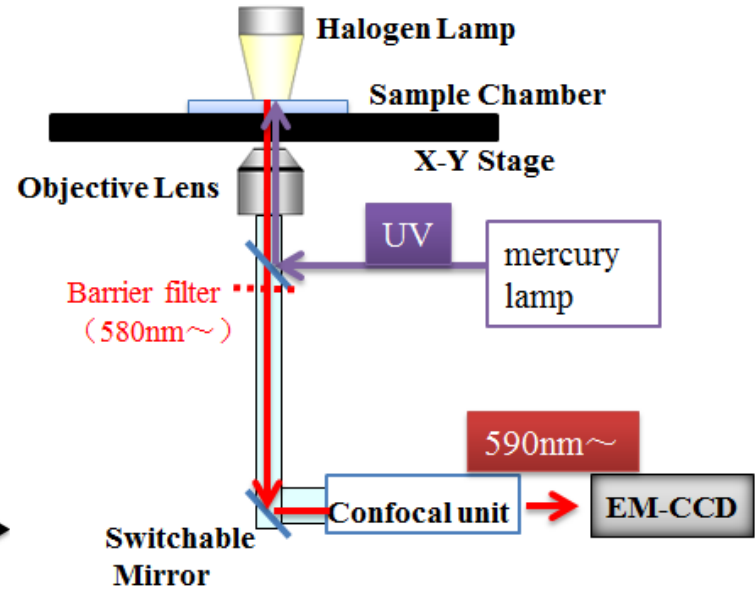

(b)

Fig. 9 FRET experiment system. (a) Overlap of the UV absorption spectrum of CellMask ${ }^{\mathrm{TM}}$ with the fluorescence emission spectrum of Lumidot ${ }^{\mathrm{TM}} \mathrm{CdSe} / \mathrm{ZnS}$ 510. (b) Experimental system for FRET observation.

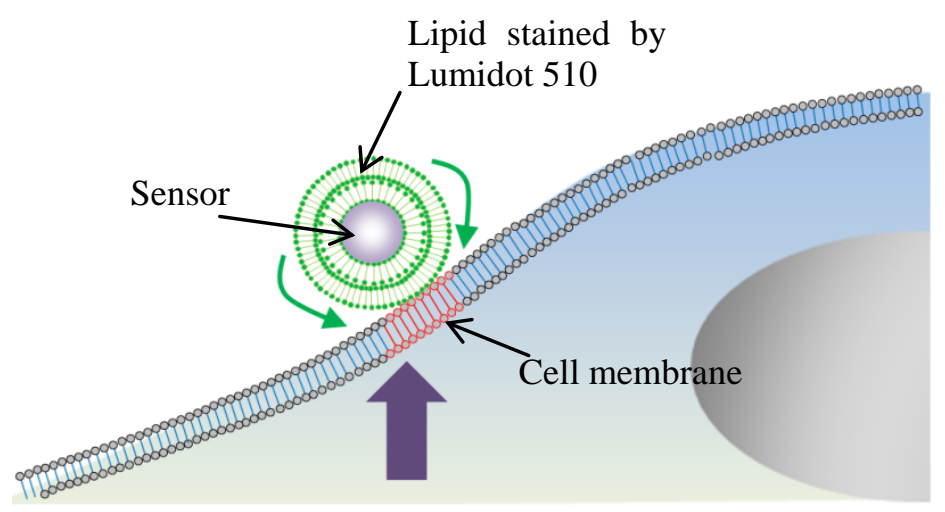

Fig. 10 Schematic design of FRET experiment between a single sensor and the cell membrane. 


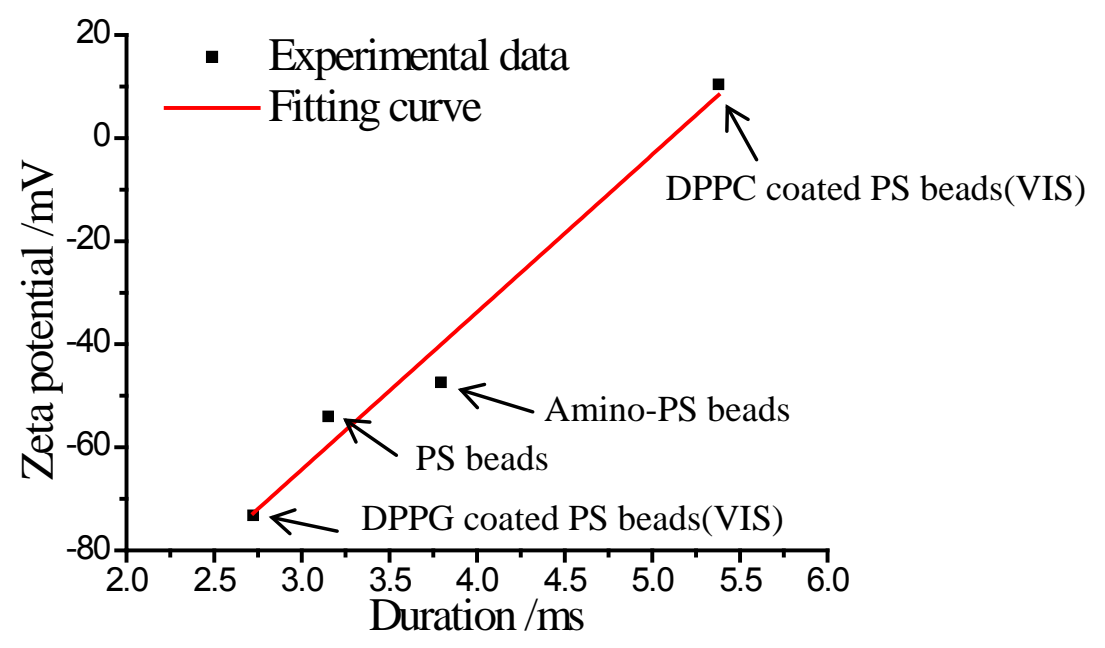

Fig. 11 Calibration result of the relationship between zeta potential and duration of particles passing through the pore in particle charge analysis.

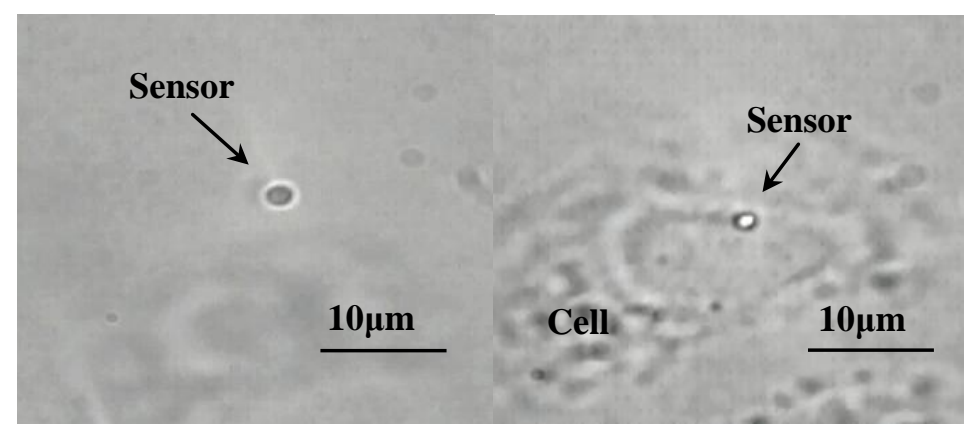

(a)

(b)
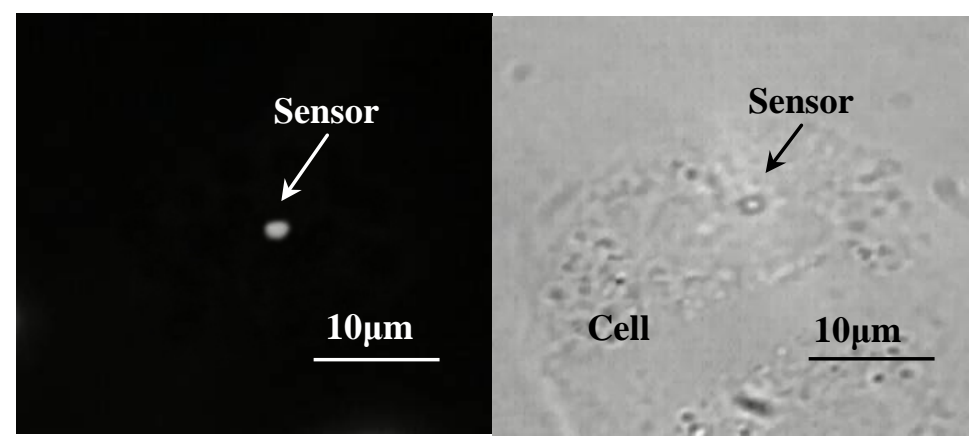

(c)

(d)

Fig. 12 Selective adhesion of a single sensor to the cell membrane by photo-isomerization. (a) A single sensor trapped by optical tweezers. (b) Transfer of the sensor to the surface of an MDCK 
cell. (c) UV illumination of the sensor for adhesion. (d) Sensor adhesion on the cell surface after UV illumination.

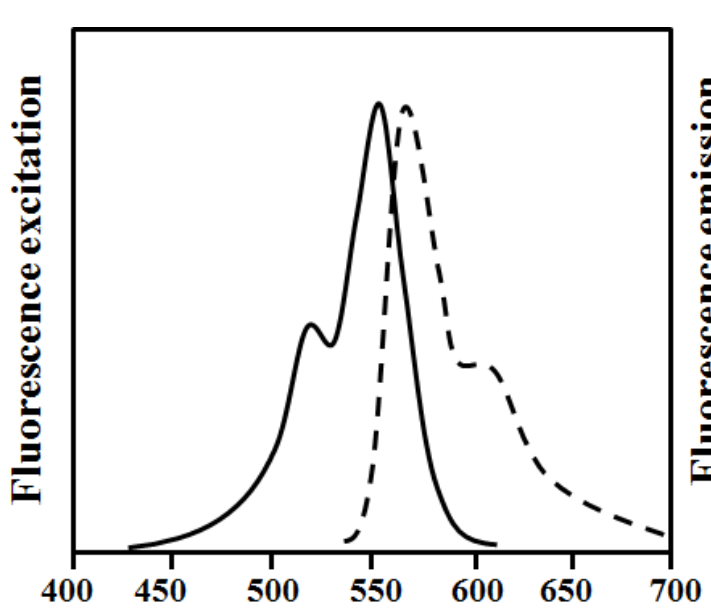

(a)

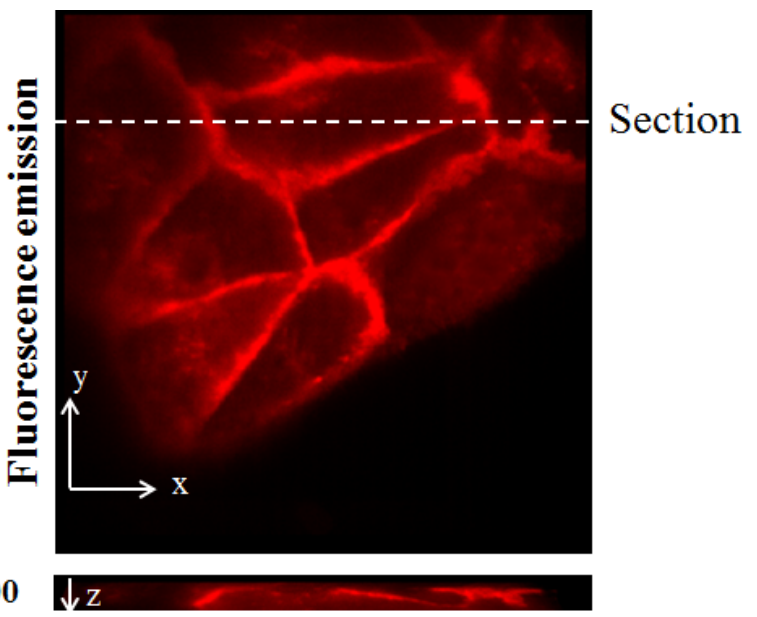

(b)

Fig. 13 Fluorescence image of cell membrane stained by CellMask ${ }^{\mathrm{TM}}$. (a) The fluorescence emission spectra of CellMask ${ }^{\mathrm{TM}}$ Orange plasma membrane stains. (b) 3D fluorescence image of cell membrane (ex. $561 \mathrm{~nm})$.

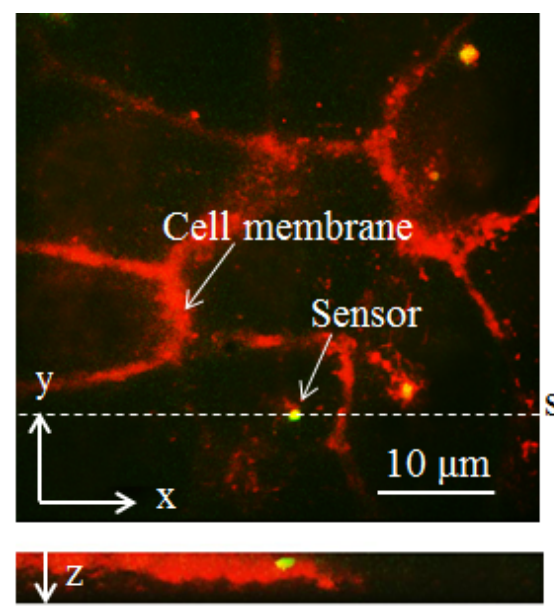

(a)

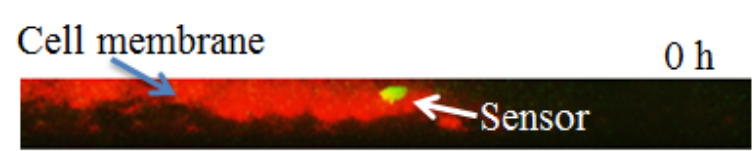

Cell membrane

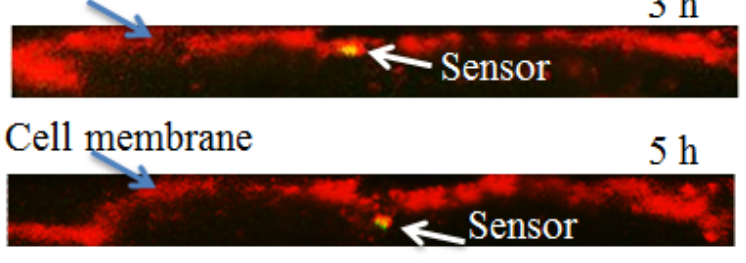

(b)

Fig. 14 Injection result based on lipofection without vibration stimulus on the sensor. (a) 3D fluorescent image of a single sensor $(\Phi 1 \mu \mathrm{m})$ adhered to the cell membrane before injection. (b) 
Process of injecting the sensor observed by 3D fluorescent image taken at different times after sensor adhesion (0 h, $3 \mathrm{~h}, 5$ h).

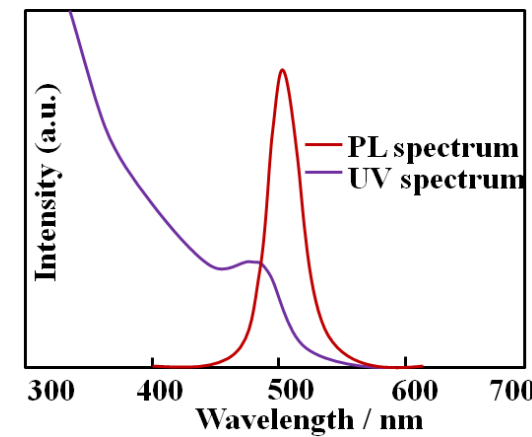

(a)

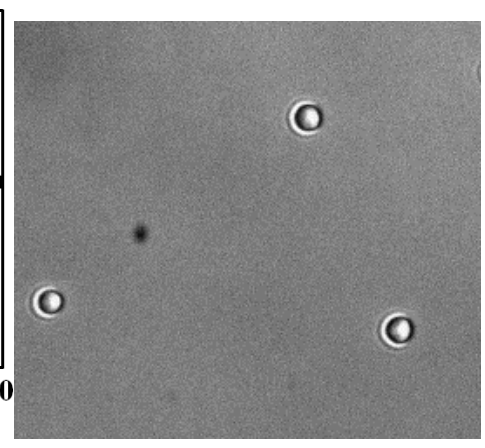

(b)

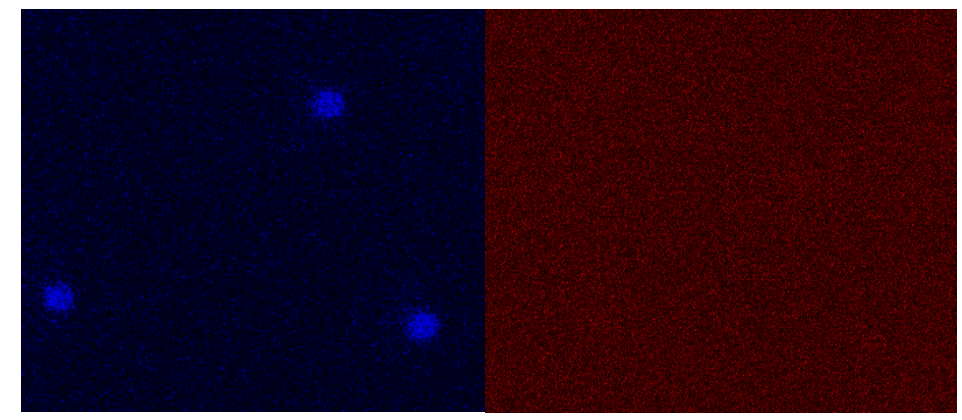

(b)

(d)

Fig. 15 Observation of the sensor $(\Phi 5 \mu \mathrm{m})$ stained by quantum dots. (a) Typical absorption and photoluminescence spectra of quantum dots (Lumidot ${ }^{\mathrm{TM}}$ CdSe-ZnS 510, ex. 366 nm, em. 510 nm). (b) Optical image of the sensor observed under bright-field. (c) Fluorescent image of the sensor (ex. 366 nm, em. 420 nm). (d) Fluorescent image of the sensor (ex. 366 nm, em. 590 $\mathrm{nm})$. 


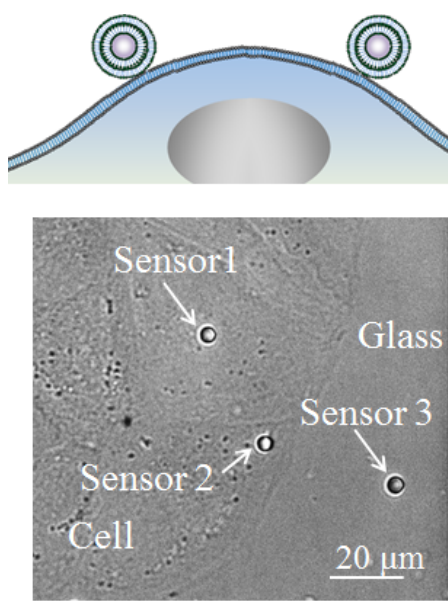

(a)

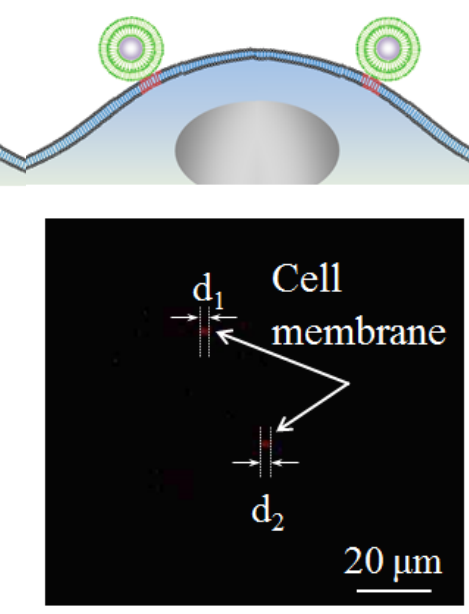

(b)
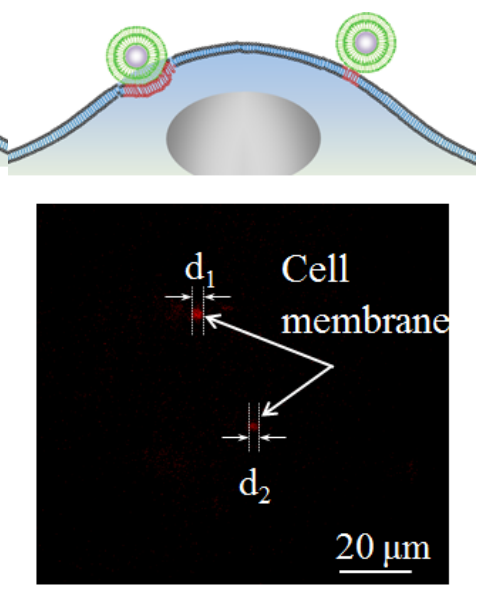

(c)

Fig. 16FRET experiment. (a) Bright-field image of the sensors. Sensor 1 and Sensor 2 adhered to the cell surface and Sensor 1 was later vibrated by optical tweezers. Sensor 3 adhered to the glass dish. (b) Fluorescent image of the cell membrane excited by FRET before vibration experiment. (c) Fluorescent image of the cell membrane excited by FRET after vibration experiment. $d_{1}$ and $d_{2}$ are the diameters of the fluorescent membrane in the positions of Sensor 1 and Sensor 2, respectively. 


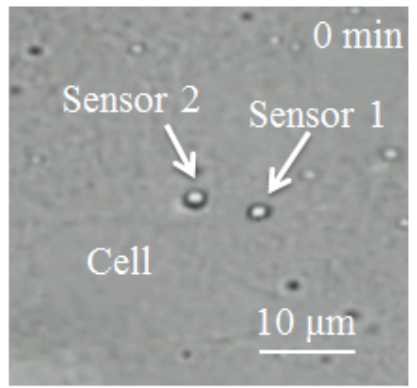

(a)

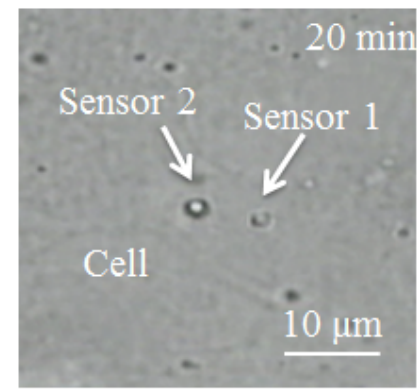

(b)

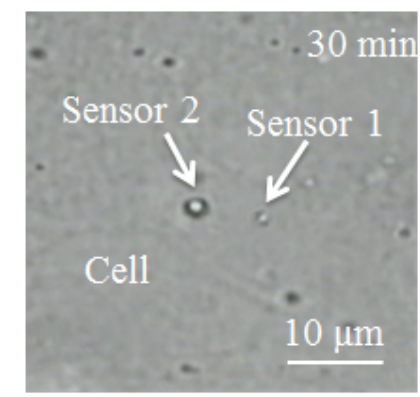

(c)

(d)

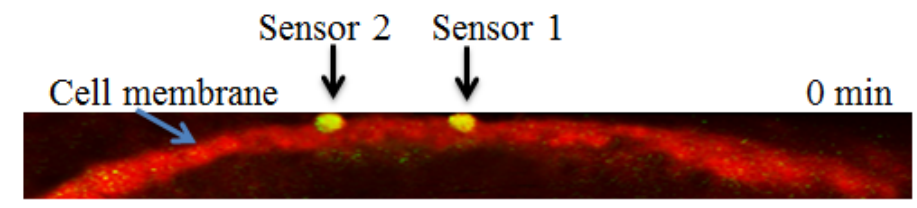

(e)

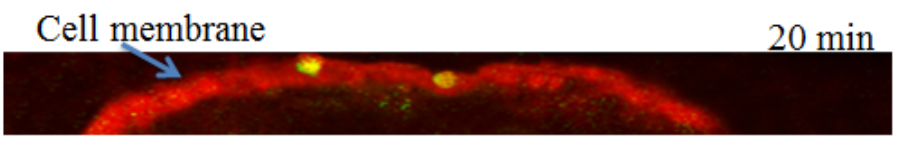

(f)

Cell membrane -1 $30 \mathrm{~min}$

Fig. 17 Local injection of individual sensor into a single cell with vibration stimulus applied by optical tweezers. $(\mathrm{a}-\mathrm{c})$ Bright-field images of sensors taken at different times: before applying the stimulus, after applying vibration for $20 \mathrm{~min}$ on Sensor 1, and $10 \mathrm{~min}$ after vibration, respectively. (d-f) 3D fluorescentimages (in multi-channels of red and green) taken at different times: before applying the stimulus, after applying vibration for 20 min on Sensor 1, and 10 min after vibration, respectively. Green channel: ex. 488 nm, em. 510 nm, Red channel: ex. 561 nm, em. $590 \mathrm{~nm}$. 